\title{
Reply to the comments of Cebesoy et al.: Cerclage cable in fracture: frustration or necessity?
}

\author{
Thorben Müller • Tobias Topp • Christian Kühne • \\ Gershon Gebhart • Steffen Ruchholtz • Ralph Zettl
}

Received: 20 February 2011 /Accepted: 22 February 2011 /Published online: 11 March 2011

(C) Springer-Verlag 2011

Thank you very much for the helpful comments in your letter to the editor.

We are glad that our manuscript [1] found broad interest and we are always disposed to share and discuss experiences.

Principally we have similar views. Of course, we use modern implants for osteosynthesis, and that is why the Gamma III nail is standard in our department. Biomechanical evaluations showed very satisfying results for Gamma III nails.

In our opinion the reason for failure of osteosynthesis in complicated subtrochanteric fracture is neither caused by the anatomical crossing from diaphysis into metaphysis in the fracture zone, nor by the lesser blood supply as it is described by Cebesoy et al. We think the persistent instability of the fragments, due to the fracture morphology in subtrochanteric fracture, represents the main difficulty (lack of medial hinge, insufficient reduction). Of course accurate reduction and the appropriate knowledge of the implants and the characteristics of the fracture together with operative experience are requirements for successful osteosynthesis. However, the correct siting and reduction of the fragments can be very challenging in spite of sufficient traction. Sometimes adequate reduction only succeeds by a mini open approach. This problem of the rare cases with unsuccessful reduction has not been solved by any implant to this day and is independent of operative expertise.

T. Müller $(\bowtie) \cdot$ T. Topp $\cdot$ C. Kühne $\cdot$ G. Gebhart $\cdot$ S. Ruchholtz $•$ R. Zettl

Department of Trauma-, Hand- and Reconstructive Surgery,

University of Giessen and Marburg,

Marburg, Germany

e-mail: thmuelle@med.uni-marburg.de
Our study design is not applied to the simple, well reduced fractures, but rather to the oblique and spiral subtrochanteric fractures. If it is necessary we think these fractures can be treated better by an optional cerclage after mini open approach and reduction by a clamp without compromising the blood supply significantly (Fig. 1).

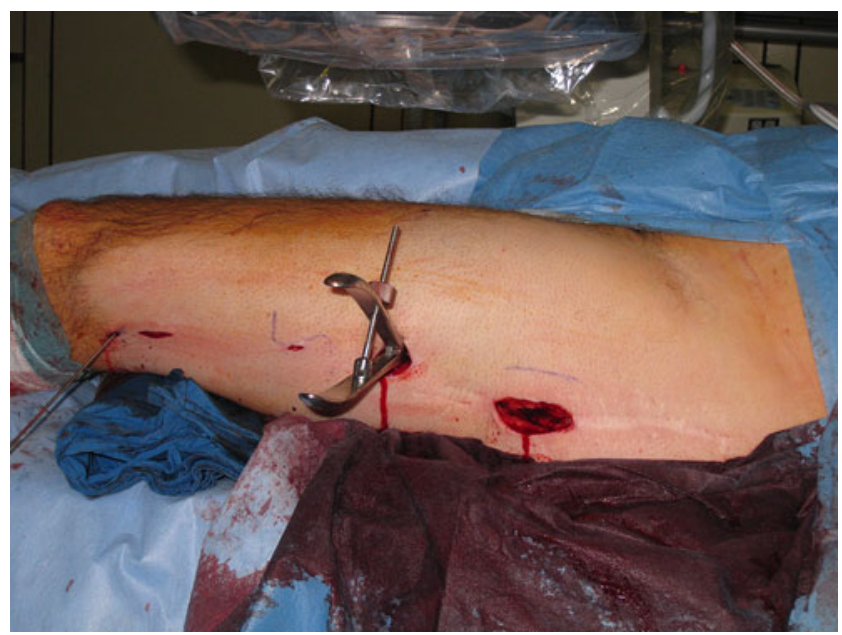

Fig. 1 Picture of surgical example with mini open approach and reduction by a clamp before cerclage implantation

\section{Reference}

1. Muller T, Topp T, Kühne CA, Gebhart G, Ruchholtz S, Zettl R (2011) The benefit of wire cerclage stabilisation of the medial hinge in intramedullary nailing for the treatment of subtrochanteric femoral fractures: a biomechanical study. Int Orthop. doi:10.1007/ s00264-010-1204-4 